\title{
Miniaturized E-Shaped PIFA Antenna for Wideband Implantable Biomedical Applications
}

\author{
F. Gozasht ${ }^{1}$ Md. Delwar Hossain ${ }^{2} \quad$ Ananda Sanagavarapu Mohan ${ }^{3}$
}

\begin{abstract}
This paper presents an implantable miniaturised printed E-shaped PIFA antenna for biomedical applications at 2.4 GHz. The design and measured results for the proposed antenna suitable for implanting either in the arm or under the chest are presented. Simulations are conducted using both homogeneous and inhomogeneous phantoms to help to design the proposed antenna. The measured results are obtained by immersing a prototype of the proposed antenna in a phantom that consists of meat-mixture. The antenna is fed by a coaxial probe and optimizes the placement of shorting pin to obtain superior performance. The antenna occupies a volume less than $1 \mathrm{~cm}^{3}$ without the biocompatible insulation and its size reduction is about $60 \%$ compared to the standard E-shaped patch antenna reported in the literature.
\end{abstract}

\section{INTRODUCTION}

The last decade has seen tremendous growth in wireless communication systems and their application in biomedical engineering. The design of implantable biomedical devices that help to improve people's health care, wellbeing and quality of life, has attracted a lot of interest. As a result a number of antennas and systems for implanting into human body have been proposed [1-4].

The study of the communication devices used for establishing a wireless link between the implanted device and the external station becomes essential when considering biomedical applications. The implanted system must fulfill miniaturization and good radiation performance. An antenna can be designed in either air or the dielectric of the human body. For the best performance from the implanted antenna when it is placed inside the human body, it must be designed taking the effects of the body and the environment in which it is expected to operate into consideration [5]. Thus, the dielectric properties of the biological tissues such as skin, fat and bone must be considered for optimum performance. In practice, they can be tested using tissue equivalent liquids and mimicking gels [2] or animals [6].

In this study, an implantable E-shaped antenna with shorting pin between the patch and the ground plane with an aim to miniaturize the size of the antenna is proposed for operation in $2.4 \mathrm{GHz}$ ISM (Industrial Scientific Medical) band. We also demonstrate the effectiveness of the shorting pin technique for size reduction of the $\mathrm{E}$ shaped PIFA antenna. The proposed antenna is compact in size and has a measured return loss of $-10 \mathrm{~dB}$ over the chosen ISM band.

\section{Antenna Design}

Medical wireless applications such as implanted devices and on-body sensors require an antenna to transmit and receive electromagnetic signals from the human body. Hence it is essential to design an efficient implanted antenna for reliable communication.

Here, we chose an E-shaped printed PIFA antenna as it can provide significantly larger bandwidths than conventional patch antennas [7]. The design is carried in two steps. First, the implantable E-shaped antenna with a shorting post was designed to operate in the $2.4 \mathrm{GHz}$ ISM band in free space. Second a single layer superstrate is added to the antenna which will then be immersed inside multi-layered humantissue mimicking phantoms to resonate at $2.4 \mathrm{GHz}$.

The feeding structure of the E-shaped antenna consists of coaxial feeding and a shorting pin with $50 \Omega$ impedance. Impedance matching of the Eshaped antenna to $50 \Omega$ is obtained by tuning the spacing between the post and the feed. The configuration of proposed antenna in air is shown in Figure 1.

\subsection{The effect of shorting post}

The existing E-shaped antenna available in the literature that operates at $2.4 \mathrm{GHZ}$ has larger dimensions with volume of $31.5 \mathrm{~cm}^{3}$ [7]. In this paper we reduce the size of the proposed E-shaped antenna by employing the shorting post.

The shorting post perturbs the field in a complex manner and cannot be described simply as a disturbance effect. However, the effect on the input impedance can be described by a shunt inductor. The pins can be used to modify the impedance of the antenna structure. The overall size of the antenna can

\footnotetext{
${ }^{1}$ Centre for Health Technologies, Faculty of Engineering and IT, University of Technology Sydney, Sydney, Australia;

E-mail: Farhad.gozasht@student.uts.edu.au

${ }^{2}$ Centre for Health Technologies, Faculty of Engineering and IT, University of Technology Sydney, Sydney, Australia; E-mail: Md.D.Hossain@student.uts.edu.au

${ }^{3}$ Centre for Health Technologies, Faculty of Engineering and IT, University of Technology Sydney, Sydney, Australia; E-mail: Ananda.Sanagavarapu@uts.edu.au, tel.: +610295142447
} 
be reduced when the pins are installed in proper locations which depend on the resonant modes [9].

The distributed parameters R, C, L and G and the length of the transmission line $(\mathrm{h})$ can be used to analyze the effect of pins. The values of $R$ and $G$ can be neglected. The series inductance $\mathrm{L}$ and the shunt capacitance $\mathrm{C}$ depend on the number of pins used, the separation between pins (d), their radii (a), the permittivity $\varepsilon$ and the permeability $\mu$ and thickness ' $h$ ' of the substrate. L and C are given by [10]

$$
\begin{aligned}
& L=\left(\frac{h \mu}{\pi}\right) \cosh ^{-1}\left(\frac{d}{2 a}\right) \\
& C=(h \pi \varepsilon) \cosh ^{-1}\left(\frac{d}{2 a}\right)
\end{aligned}
$$

The resulting reactance of the shorting pins will be either inductive or capacitive depending on the values of $\mathrm{L}$ and $\mathrm{C}$ : normally $\mathrm{L}$ and $\mathrm{C}$ increase when the thickness $\mathrm{h}$ of the substrate is increased. The resonant frequency of the antenna will decrease if the reactance of the shorting pin is inductive, while capacitive reactance will increase the resonant frequency [10]. The maximum size reduction occurs when only a single pin is used. When the feed location is close to the pin, at the resonant frequency, the input resistance decreases making it difficult to match it to a $50 \Omega$ coaxial feed.

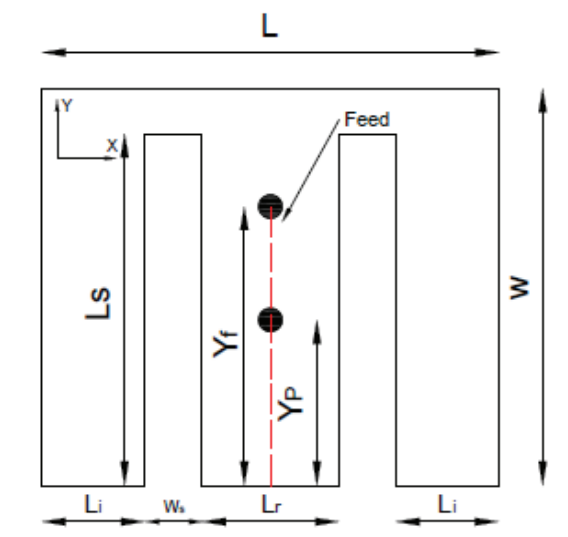

Figure 1: Geometry of E-shaped patch antenna.

To significantly reduce the size of the E-shaped antenna, a single shorting pin between the ground plane and the patch was used and its location was varied in a trial and error manner to obtain resonance at frequencies of interest. Thus, our proposed antenna designed to operate inside human body has a volume of $0.5 \mathrm{~cm}^{3}$ which much smaller than the published antenna [7] Use of the shorting pin at appropriate location, could help to reduce the volume of the antenna very significantly. The dimensions of the proposed PIFA are $\mathrm{L}=19 \mathrm{~mm}, \mathrm{~W}=17 \mathrm{~mm}$ and $\mathrm{h}=1.6 \mathrm{~mm}$ (volume of $0.5 \mathrm{~cm}^{3}$ ) which resonates at 2.4
$\mathrm{GHz}$ inside human tissue mimicking phantom. The proposed antenna is printed on FR4 substrate for testing its performance. When a superstrate is added to the antenna, its volume increases.

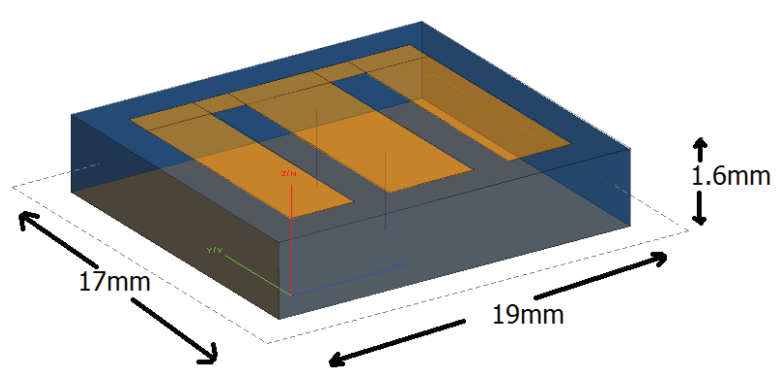

Figure 2: Geometry of E-shaped antenna on FR4 substrate.

We have observed that resonance occurs at two different frequencies when a shorting pin is positioned close to the feeding point as shown in Figure 5. This feature can be used to tune it to operate at multiple frequency bands if needed. To achieve wideband antenna performance, it is necessary to modify the feed and pin positions. To obtain the appropriate feeding position, we employ numerical experiments using FEKO $^{\mathrm{TM}}$ [12] to determine the antenna input impedance match. Our investigations have indicated that, for the same antenna size, the antenna can also made to resonate at $950 \mathrm{MHz}$ band by adjusting the position of the shorting pin.

\subsection{Simulations inside Human Body Model}

The characteristics of the antenna are affected by the skin thickness and its dielectric properties since implanted antennas are typically located under the body skin [1]. However, in some cases, antenna will be placed under the body fat and/or muscle e.g., in inter-body communication applications[2]. Therefore, the properties of fat and muscle should also be taken into account.

In simulations, for human body model, we have used well-known cylindrical and layered phantoms, as shown in Figure 3(a)-(b). In numerical simulations, we have chosen the length of the phantom to be $100 \mathrm{~mm}$. Also, we assume that the antenna is embedded into the fat layer of the phantoms at a depth of $2.0 \mathrm{~mm}$ from the skin surface [1]. Cylindrically shaped multi-layered phantom was used for simulations to represent implantation inside the human arm [1]. The average thicknesses of each layer in the cylindrical structure are as shown in Figure 3.

We also employed a layered rectangular block phantom for simulations to represent antenna implantation under human chest. The antenna was 
positioned in the fat under the skin at $2 \mathrm{~mm}$ distance from the skin surface, as shown in Figure 3 (c). For simulations, we used the dielectric properties of skin, fat and muscle as given in Table 1 [11]. Using the simulations, the dimensions of the antenna are chosen.

\begin{tabular}{|c|c|c|c|c|}
\hline Tissue & \multicolumn{2}{|c|}{ Permittivity } & \multicolumn{2}{c|}{ Conductivity s/m } \\
\hline & $950 \mathrm{MHz}$ & $2.4 \mathrm{GHz}$ & $950 \mathrm{MHz}$ & $2.4 \mathrm{GHz}$ \\
\hline Muscle & 54.9 & 52.7 & 0.96 & 1.73 \\
\hline Skin & 41.1 & 38 & 0.88 & 1.46 \\
\hline Fat & 5.45 & 5.28 & 0.05 & 0.10 \\
\hline
\end{tabular}

Table 1: Dielectric properties of human tissues

The PIFA is composed of a single metallic layer and is printed on a side of a FR4 substrate having a dielectric constant of 4.7 , loss tangent of 0.0038 , and thickness of $1.6 \mathrm{~mm}$. The size of the proposed antenna is $19 \times 17 \times 1.6 \mathrm{~mm}$, which is quite appropriate for body implanted wireless system. The metallic layer is covered by FR4 superstrate with the same specifications. The simulation results have been carried out by $\mathrm{FEKO}^{\mathrm{TM}}$ [12].

\section{Measurements using Phantoms}

In order to measure the performances of the antenna inside a human body, a body equivalent phantom is required [8]. In the literature, for multiband operation, pork leg [8] and skin gel [1] were proposed. However, skin gel can only be used for one special band, making it quite inconvenient for verifying the multiband antenna performance. Further, the relative permittivity and conductivities of pork leg is higher than the human tissue.

Hence, we have used a mixture of lean pork and beef to obtain a mixed-meat phantom. The dielectric constant and conductivity of the mixed-meat phantom have been measured keeping it in a container having a volume of $120 \times 120 \times 45 \mathrm{~mm}^{3}$ by using dielectric assessment probe kit from SPEAG and Agilent E5071C network analyser. The measured dielectric properties of the mixture agree closely with properties of human tissues.

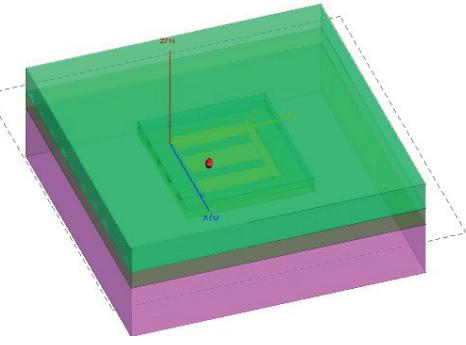

(a) Layered Block model

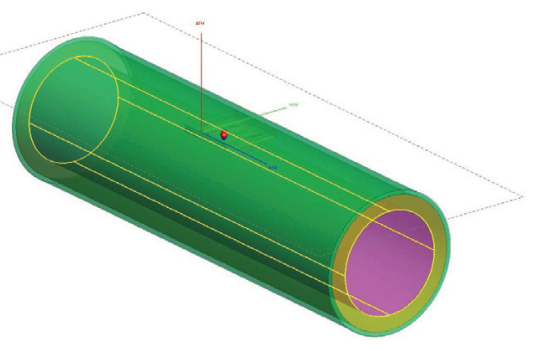

(b) Layered cylindrical model

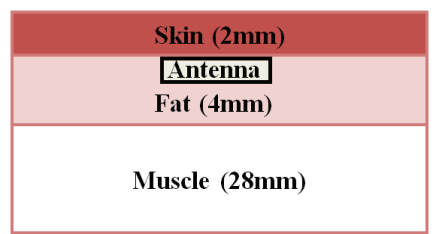

(c) Layer dimensions for simulation model.

Figure 3: Simulation models of human body phantoms.

Figure 4 shows measurement setup and the prototype of E-shaped PIFA antenna on FR4 substrate. The simulated results on return loss for the proposed antenna when placed inside homogeneous and multi-layered phantoms are shown in Figures 5 and 6.We have also compared measured data using meat-mixture-phantom with simulation results in the same figures. The results indicate that the results are consistent.
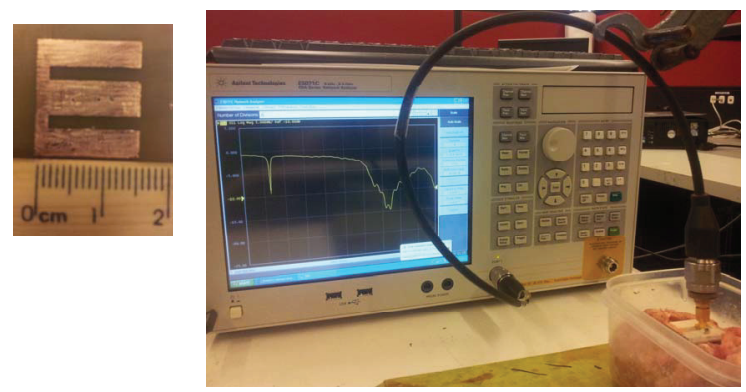

Figure 4: E-shaped antenna on FR4 substrate and Measurement set up. 
The results illustrated in Figure 5 show that the antenna has a return loss of $-30 \mathrm{~dB}$ at $2.4 \mathrm{GHz}$ and has an impedance bandwidth of $250 \mathrm{MHz}$, which is sufficient for implanted biomedical applications. The results plotted in Figure 6 also demonstrate reasonable agreement with simulations using layered phantoms.

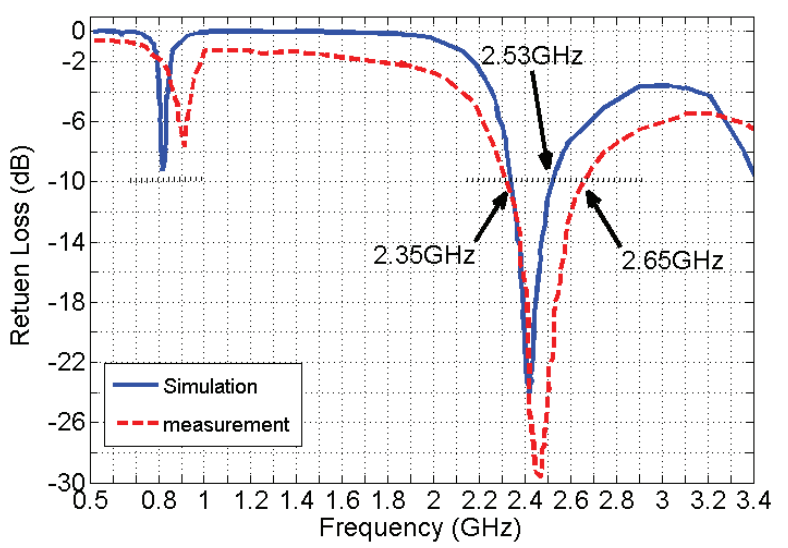

Figure 5: Measured vs. Simulation : Homogeneous phantom

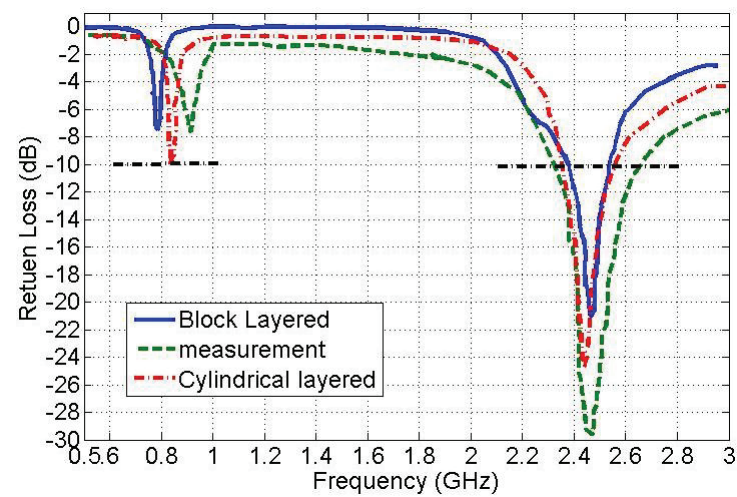

Figure 6: Measured vs. Simulation Block and cylindrically Layered phantoms

\section{Conclusions}

This study describes a miniaturized E-shaped implantable antenna of size $19 \times 17 \mathrm{~mm}^{2}$ with a shorting pin. The antenna obtains a $250 \mathrm{MHz}$ bandwidth at a centre frequency of $2.4 \mathrm{GHz}$. By varying the pin position, the antenna can be made to resonate at other frequencies without changing the dimensions. Simulations using block and cylindrically multi layered tissue mimicking phantoms were carried out and compared with measured results. The implantable antenna exhibits smaller size, lower return loss and wider band impedance bandwidth for use in biomedical implant communications.

\section{Acknowledgments}

The work reported in this paper is supported in-part by the Australian Research Council through a Discovery Grant DP 0773234.

\section{References}

[1] H. Lin, M. Takahashi, K. Saito and K. Ito, "Performance of Implantable Folded Dipole Antenna for In-Body Wireless Communication", IEEE Trans. Antennas Propag., vol. 61, No. 3, March 2013.

[2] T. Karacolak, A. Hood and E. Topsakal, "Design of a dual-band implantable antenna and development of skin mimicking gel for continuous glucose monitoring", IEEE Trans. Microw. Theory Tech., vol. 56, No. 4, April 2008.

[3] Y. Rahmat-Samii and J. Kim, "Implanted Antennas in Medical Wireless Communications", Morgan \& Claypool Publishers, 2006.

[4] M. Scarpello, et.al., "Design of an implantable slot dipole conformal flexible antenna for biomedical applications", IEEE Trans. Antennas Propag., Vol. 59, No. 10, October 2011.

[5] L. Huang, M. Ashoueil and F. Yazicioglu, "UkraLow Power Sensor Design for Wireless Body Area Networks: Challenges, Potential Solutions, and Applications", Int. J. of Digital Content Tech. and its Applications, vol. 3, Number 3, Sep. 2009.

[6] T. Karacolak, R. Cooper, J. Butler, S. Fisher and E. Topsakal, "In Vivo Verification of Implantable Antennas Using Rats as Model Animals" IEEE Antennas Wireless Propag. Lett., Vol. 9, 2010.

[7] F. Yang, X. Zhang, X. Ye and Y. Rahmat-Samii, "Wide-band E-Shaped patch antennas for wireless communications", IEEE Transactions on antennas and propagation, vol.49, No.7, July 2001.

[8] F. Huang, et.al., "Rectenna application of miniaturized implantable antenna design for triple-Band biotelemetry communication", IEEE Trans. Antennas Propag., vol.59, No.7, 2011.

[9] B.M. Alarjani, J.S. Dahele, "Feed Reactance of Rectangular Microstrip Patch Antenna with Probe Feed", Electron. Lett., vol.36, No.5, pp. 388-390, March 2000.

[10] M. Sanad, "Effect of the Shorting Posts on Short Circuit Microstrip Antennas", APS Int. Symp. AP-S. Digest, vol.2, pp. 794-797, 1994.

[11] Dielectric Properties of Body Tissues (IFAC), Available: http://niremf.ifac.cnr.it/tissprop/

[12] $\mathrm{FEKO}^{\mathrm{TM}}$, EM Software \& Systems, http://www.feko.info 